University of Windsor

Scholarship at UWindsor

\title{
Theory of detection of angular momentum states in Rydberg atoms using half-cycle pulses
}

Chitra Rangan

University of Windsor

R.J.A. Murray

Follow this and additional works at: https://scholar.uwindsor.ca/physicspub

Part of the Physics Commons

\section{Recommended Citation}

Rangan, Chitra and Murray, R.J.A.. (2005). Theory of detection of angular momentum states in Rydberg atoms using half-cycle pulses. Physical Review A - Atomic, Molecular, and Optical Physics, 72 (5), 053409-1-053409-4.

https://scholar.uwindsor.ca/physicspub/5

This Article is brought to you for free and open access by the Department of Physics at Scholarship at UWindsor. It has been accepted for inclusion in Physics Publications by an authorized administrator of Scholarship at UWindsor. For more information, please contact scholarship@uwindsor.ca. 


\title{
Theory of detection of angular momentum states in Rydberg atoms using half-cycle pulses
}

\author{
C. Rangan and R. J. A. Murray \\ Department of Physics, University of Windsor, ON, N9B 3P4, Canada
}

(Received 16 June 2005; published 17 November 2005)

\begin{abstract}
The creation and detection of specific angular momentum states in Rydberg atoms is a significant advance in quantum-information processing. Detection of high angular momentum states in alkali-metal atoms is difficult because they are nearly-degenerate in energy. We present theoretical results on the detection of such states using terahertz-frequency half-cycle pulses (HCPs). Calculations are done for $n=15, \ell=0, \ldots, 14$ states in hydrogen and cesium using a grid-based pseudopotential method. Our results indicate that HCP redistribution is a reliable method of distinguishing angular momentum Rydberg states.

DOI: 10.1103/PhysRevA.72.053409 PACS number(s): 32.80.Qk, 32.80.Rm, 32.60.+i, 03.67.-a
\end{abstract}

\section{INTRODUCTION}

Rydberg atom wave packets have been used to successfully store and retrieve quantum information [1]. Recent work has demonstrated quantum-information processing (QIP) in one coordinate of a Rydberg atom - the radial wave packet [2]. Indeed, the inversion-about-mean operation of the quantum search algorithm [3] can be performed efficiently in a radial wave packet database $[4,5]$.

A significant advance in Rydberg atom QIP can be made if information can be manipulated in two coordinates, for example both in radial and in angular coordinates. Such a capability will facilitate more complex processes such as those required for Shor's factoring algorithm [6]. In general character, this requirement is comparable to other singleparticle correlated data registers such as using vibrational and rotational quantum numbers in molecules [7], using symmetry and phase eigenstates of the Morse oscillator [8], or using photon number states and different modes in cavity photons [9].

In a recent paper [10], we proposed an interferometric scheme to produce nearly pure angular momentum states in Stark Rydberg wave packets. One of the challenges of this scheme is the reliable detection of high-angular-momentum states. In this paper, we propose a scheme for detecting highangular-momentum states-their population redistribution using a half-cycle pulse (HCP). We show that the redistributed population gives a clear signature of the initial angular momentum states. This work provides a theoretical complement to the recent experimental results in Ref. [11], that demonstrate the effect of HCPs on angular momentum states. However, the above experiment is sufficiently different from this proposed scheme that quantitative comparisons cannot be made. Possible challenges in the experimental implementation of our proposal are discussed.

\section{DETECTING ANGULAR MOMENTUM COMPONENTS IN STARK WAVE PACKETS}

In Ref. [10], it was shown that nearly pure angular momentum states can be produced in Stark wave packets at target times using ultrafast pulses. However, in an external electric field, the angular momentum is not a good quantum number. For typical experimental fields (a few hundred $\mathrm{V} / \mathrm{cm})$ and principal quantum number $(n \simeq 30)$, the angular momentum precession time is of the order of a few hundred picoseconds. Therefore, a first requirement of a detection scheme is for it to be faster than the angular momentum precession time.

Second, the redistributed population must be such that it can be measured easily by methods such as field ionization. This requires that the population be distributed preferably over several principal quantum number manifolds. The bandwidth of the redistributing field must be of the order of a terahertz. Finally, the detection scheme must be sensitive to the initial angular momentum.

A promising method that satisfies all these requirements is the redistribution of Rydberg state population using a terahertz half-cycle pulse. A typical HCP has a full width at half maximum of roughly $1 \mathrm{ps}$, which is much shorter than the angular momentum precession time of the wave packet. Both the HCP bandwidth and the central frequency are of the order of a terahertz. This ensures that the Rydberg state population will be redistributed over several principal quantum number $n$ manifolds.

The interaction of a short HCP with a Rydberg electron can be modeled as an impulsive kick (ignoring the free evolution that occurs on a larger time scale). This "impulse approximation" is valid when the width of the strong positive lobe of the half-cycle pulse is shorter than the Kepler orbital period of the wave packet. The long, weak negative tail of the HCP does not perturb the Rydberg state population. Earlier time-dependent calculations [2] show that the impulse model of a half-cycle pulse is a very good approximation to using the exact temporal form of its electric field.

The impulsive interaction of the HCP with a Rydberg electron is written as the operator $\exp (i Q z)$. Writing the position operator in spherical coordinates, and using the properties of spherical harmonics, it is clear that the HCP strongly couples angular momenta, and can be expected to be sensitive to the initial angular momentum of the Rydberg state. In the next section, we present numerical results that indicate that the HCP is indeed a reliable detector of angular momentum states in the Rydberg atom. 


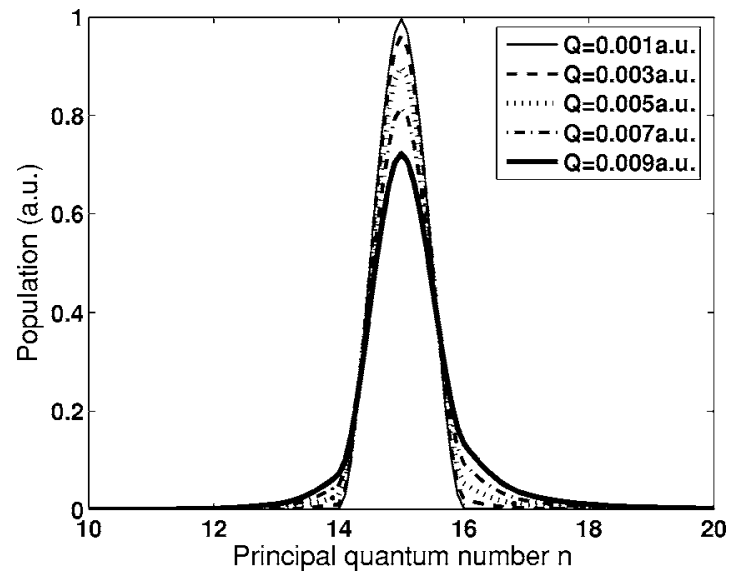

FIG. 1. Population redistribution of $|n=15, \ell=0\rangle$ state in hydrogen by a half-cycle pulse. As the peak strength of the HCP field increases, the population is redistributed over an increasing number of principal quantum number manifolds centered about the initial state.

\section{RESULTS AND DISCUSSION}

In this section, we present numerical results of the interaction between an impulsive HCP and angular momentum states of hydrogen and cesium. The field-free eigenstates are calculated using a grid-based pseudopotential method [12], and the HCP is modeled as an impulsive momentum kick $\exp (i Q z)$ to the electron, where $Q$ is the strength of the kick. The experiments are typically done on $n \simeq 30$ states; however, the impulsive behavior scales extremely well with principal quantum number $n$ and $Q$ [12]. Therefore, the calculations of final-state population distributions are presented for $n=15$. The parameters that are varied are the angular momentum of the initial state and the strength of the kick $Q$.

Figure 1 shows the HCP redistributed population of initial low-angular momentum states of hydrogen as a function of the HCP strength. For the $|n=15, \ell=0\rangle$ state, it is seen that the redistribution is symmetric about the initial state, and has an almost Gaussian distribution in energy. This feature is similar to the photoelectron spectra observed in charged particle collisions [13]. As the strength of the kick increases, the distribution gets wider in $n$ as expected, but the general characteristics do not change.

Figure 2 shows the HCP redistributed population of initial high-angular-momentum states of hydrogen as a function of the HCP strength. For the $|n=15, \ell=14\rangle$ state, it is seen that the redistribution is symmetric about the initial state, but the distribution changes to bimodal at higher $Q$.

Clearly, high- $\ell$ states are redistributed very differently from low- $\ell$ states by a strong HCP. The sensitivity to the initial angular momentum of these states is seen in Fig. 3. The impulse applied is fairly strong at $Q=0.01$ a.u. As noted before, the low-angular-momentum states have a single peak in the redistributed population, and the high-angularmomentum states have a bimodal pattern of redistribution. The change in behavior occurs at $\ell=n / 2$.

The bimodal structure can be explained in two ways. Quantum mechanically, the HCP couples states both lower

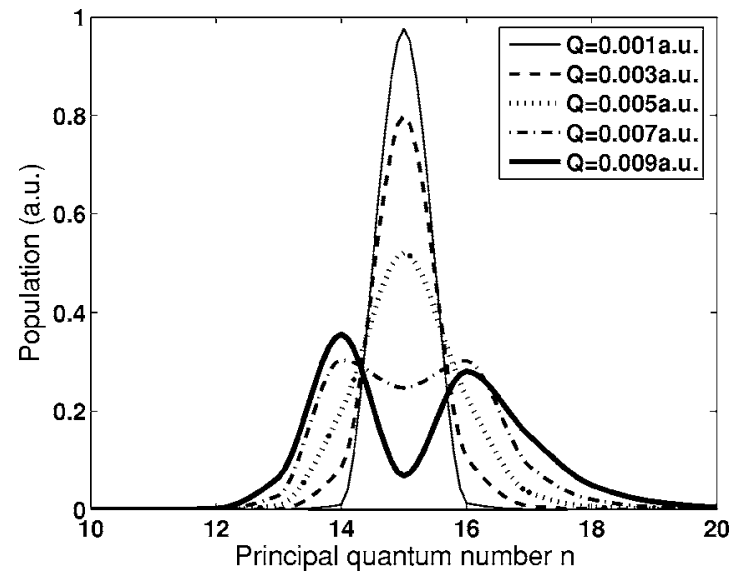

FIG. 2. Population redistribution of $|n=15, \ell=14\rangle$ state in hydrogen by a half-cycle pulse. As the peak strength of the HCP field increases, the population is redistributed over an increasing number of principal quantum number manifolds centered about the initial state. However, at large peak field strengths, the distribution becomes bimodal.

and higher $\ell$, and this causes a two-path interference in each $n$-manifold. A clearer understanding can be obtained by looking at the classical phase-space trajectories of the eigenstates. The trajectories for the $n=15, \ell=0,1, \ldots, 14$ states are well known and are shown in Fig. 4. The trajectories for the low$\ell$ states pass very near the nucleus and are closely spaced at large $r$. Those of the high- $\ell$ states do not approach the nucleus as closely, and are widely spaced at large $r$. The transition between these two patterns appears roughly at $\ell=n / 2$, where the Coulomb and the centrifugal forces are comparable to each other. As expected, the phase-space density is the highest near the classical turning points, but for the high- $\ell$ states, the difference in the density between the turning points and other locations is smaller than for the low- $\ell$ states.

The HCP in the impulsive limit provides a momentum boost to the electron. Thus, the phase space distribution of

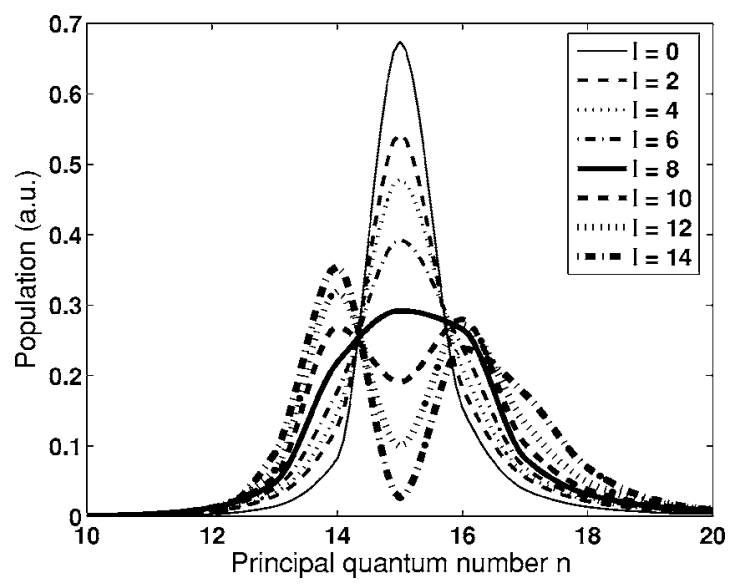

FIG. 3. Population redistribution of $|n=15, \ell\rangle$ states in hydrogen by a half-cycle pulse of strength $Q=0.01$ a.u. As the angular momentum of the initial state changes from $\ell=0$ through $\ell=n-1$, the population redistribution changes character from a single-lobe structure to a bimodal structure. The transition appears to occur around $\ell=n / 2$. 


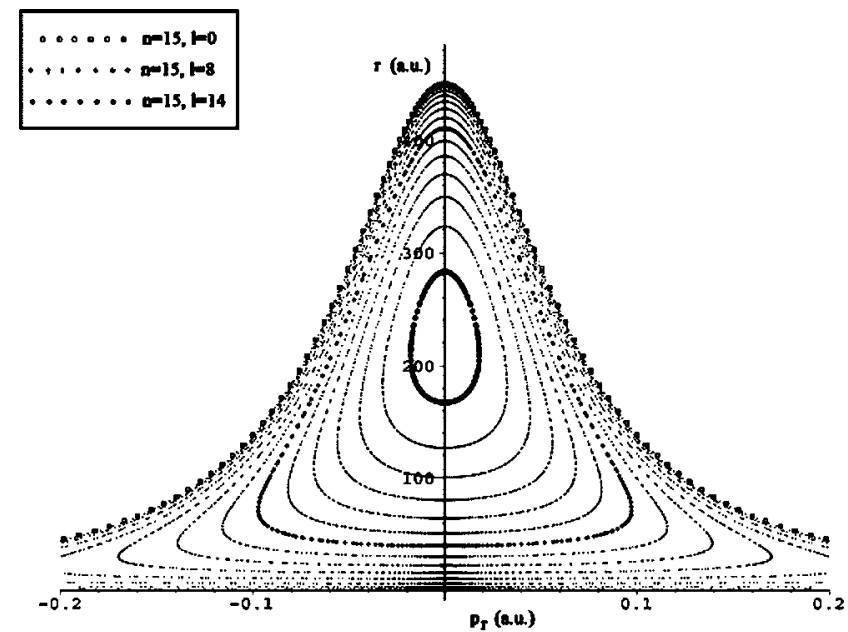

FIG. 4. Classical trajectories of a particle in a radial Coulomb potential corresponding to energies $n=15, \ell=0,1, \ldots, 14$. The transition from low- $\ell$ to high- $\ell$ behavior occurs at around $\ell=n / 2$.

the kicked state will be displaced along the momentum axis. The overlap of the displaced trajectory with the unperturbed $|n, \ell\rangle$ trajectories is a measure of the overlap of the final-state wavefunction with the eigenfunctions of the unperturbed Hamiltonian [14]. Figure 5 shows the trajectories corresponding to the $n=15, \ell=0,8,14$ states kicked by an impulsive HCP of $Q=0.01$ a.u. To aid visibility, only those trajectories corresponding to the $n=14$ and 16 states are drawn.

The trajectory of the kicked $n=15, \ell=0$ state has the largest overlap with other $n=15, \ell$ states, and to a smaller extent with the $n=14, \ell$ and $n=16, \ell$ states. Therefore the photoelectron distribution shows a symmetric distribution centered about $n=15$. The displaced phase-space trajectory has strong overlap with two $n$ manifolds, both symmetric about the initial $n$ manifold. The trajectory of the kicked $n=15, \ell=14$ state has no overlap with other $n=15, \ell$ states, but overlaps with the $n=14, \ell=13$ and $n=16, \ell=15$ states. This explains

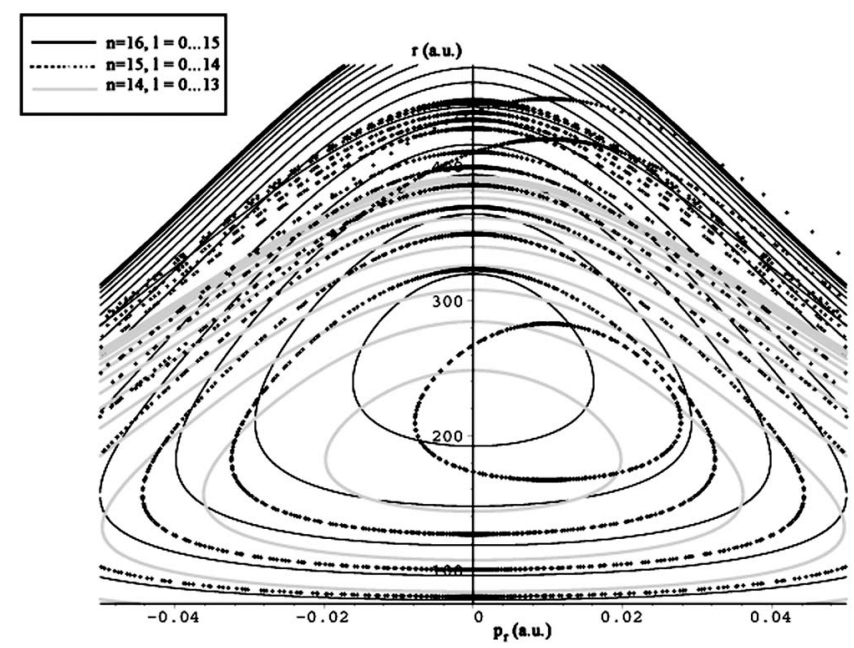

FIG. 5. Classical trajectories of a particle in a radial Coulomb potential corresponding to energies $n=14,15,16, \ell=0, \ldots, n-1$. The states $n=15, \ell=0,8,14$ are kicked by a half-cycle pulse, and are boosted by momentum $Q=0.01$ a.u.

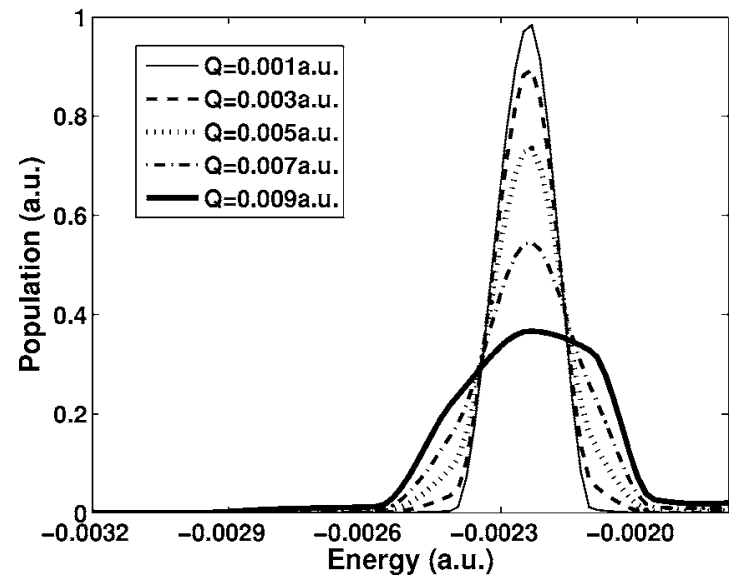

FIG. 6. Population redistribution of $|n=15, \ell=0\rangle$ state in cesium by a half-cycle pulse. Here, $n=15$ indicates the state closest to the hydrogenic $n=15$ state. As the peak strength of the HCP field increases, the population redistribution is very similar to that in hydrogen.

the dip in the photoelectron spectrum around $n=15$ and the apparent bimodal distribution. The transition between these two behaviors occurs for kicked states with $\ell=n / 2$.

Thus, a HCP can easily distinguish between states with initial $\ell$ lesser than or greater than $n / 2$. However, it probably cannot reliably distinguish between the $\ell=n-1$ and the $\ell=n-2$ states. However, even this level of sensitivity is a large advance in the detection of angular momentum states in Rydberg atoms.

To aid comparison with experiments, similar results as above are shown in cesium. In cesium, the $s$-states are degenerate with the $\ell>2$ states; and the $p$ and $d$ states are nearly-degenerate with each other, but separated from the higher- $\ell$ (hydrogenic) states [15]. In this paper, we do not use the $n^{*}$ notation. The $n=15, \ell=0$ state in cesium simply indicates the $s$ state that is closest in energy to the $n=15, \ell$ $>2$ states. Results similar to Figs. 1 and 2 in the case of cesium are shown in Figs. 6 and 7, respectively. However, the redistributed populations are plotted as a function of energy rather than principal quantum number.

In general, the redistribution in cesium is similar to that in hydrogen, except for differences because of the quantum defect. Population in the $s$ states couple via the $p$ and $d$ states to higher angular momenta; therefore the behavior of the low- $\ell$ states is similar to that of hydrogen. For the high- $\ell$ states, the $p$ and $d$ states are well removed in energy from the rest of the manifold that they do not get significantly populated. Ignoring the population in these states (which appear like zeros between the peaks in the graph), the redistributed population is again bimodal as seen in hydrogen. Thus the HCP can distinguish between high- and low-angular-momentum populations in alkali-metal atoms as well.

\section{SUMMARY}

In summary, numerical calculations indicate that the HCP redistribution of Rydberg population is a good diagnostic of initial state angular momentum composition. With a strong 


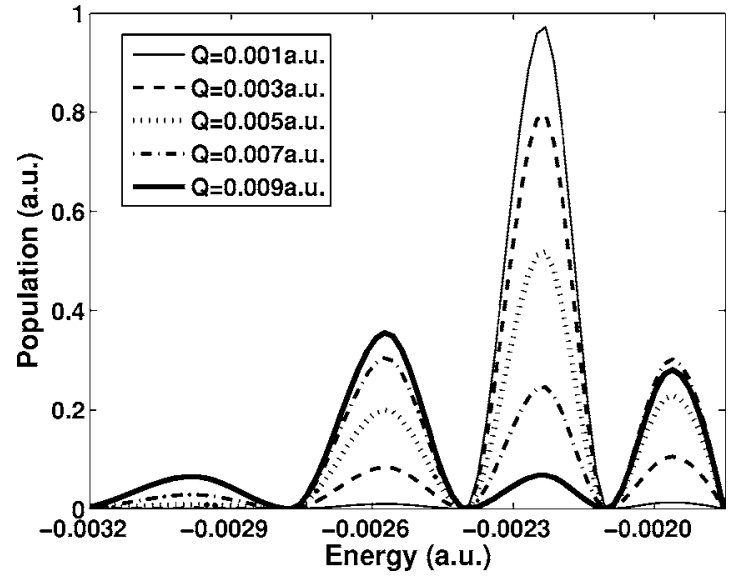

FIG. 7. Population redistribution of $|n=15, \ell=14\rangle$ state in cesium by a half-cycle pulse. The population redistribution of high- $\ell$ states with increasing $Q$ is very similar to that in hydrogen, except for the fact that the $p$ and $d$ states which are separated in energy from the rest of the manifold are not significantly populated.

$\mathrm{HCP}$, it is possible to clearly distinguish between high- and low-angular-momentum states.

Several challenges remain in experimental implementation. At high $Q$ values or peak HCP field strengths, the tail of the HCP is strong and cannot be ignored. This will probably influence the redistributed population, although in the recent experiment [11], this does not appear to be a problem. Timedependent calculations with the electro-optically sampled HCP field data can provide a stronger basis of comparison to the experiment.
This paper presents the redistribution of population from angular momentum states of a single $n$ manifold. An interesting question is: what happens when one has nearly pure angular momentum states from several different manifolds? Preliminary calculations indicate that this method can be effective for distinguishing pure- $\ell$ states from not-so-near $n$ manifolds. For states from neighboring $n$ 's, the redistributed population does not provide as good a signature of initial $\ell$ as for the single- $n$-manifold case. This can be understood by looking at the phase-space trajectories again. If the states of two neighboring $n$ 's with the same large $\ell$ are kicked, population will be transferred from one $n$ manifold into the other, thereby preventing a dip in the spectrum at large $Q$. However, if the two $n$ manifolds are separated enough that the phase-space trajectories of their states do not overlap, the HCP will be a good method of distinguishing the angular momentum states. One may then envision the production and detection of states such as $\left|n_{1} \ell_{1}\right\rangle+\left|n_{2} \ell_{2}\right\rangle$, and implement two-coordinate quantum-information processing. Therefore, the ability to distinguish angular momentum states using a half-cycle pulse is a significant advance in Rydberg atom quantum-information processing.

\section{ACKNOWLEDGMENTS}

It is a pleasure to thank Phil Bucksbaum, Santosh Pisharody, Haidan Wen, and Joel Murray at the University of Michigan for several helpful discussions, and Ravi Rau (Louisiana State University) and John Delos (College of William and Mary) for helpful comments. R.J.A.M. thanks Misha Ivanov (NRC, Ottawa, Canada) for support during his undergraduate research experience term. Our research is currently supported by NSERC, Canada.
[1] J. Ahn, T. C. Weinacht, and P. H. Bucksbaum, Science 287, $463(2000)$

[2] J. Ahn, D. N. Hutchinson, C. Rangan, and P. H. Bucksbaum, Phys. Rev. Lett. 86, 1179 (2001).

[3] L. K. Grover, Phys. Rev. Lett. 79, 325 (1997).

[4] C. Rangan, J. Ahn, D. Hutchinson, and P. H. Bucksbaum, J. Mod. Opt. 49, 2239 (2003).

[5] C. Rangan and P. H. Bucksbaum, Phys. Rev. A 64, 033417 (2001).

[6] P. W. Shor, in Proceedings of the 35th Annual Symposium on the Foundations of Computer Science, edited by S. Goldwasser (IEEE Computer Society, Los Alamitos, CA, 1994).

[7] Jos P. Palao and Ronnie Kosloff, Phys. Rev. Lett. 89, 188301 (2002).

[8] E. A. Shapiro, Michael Spanner, and Misha Yu. Ivanov, Phys.
Rev. Lett. 91, 237901 (2003).

[9] See, for example, B. Hessmo et al., Phys. Rev. Lett. 92, 180401 (2004), and references therein.

[10] H. Wen, C. Rangan, and P. H. Bucksbaum, Phys. Rev. A 68, 053405 (2003).

[11] H. Wen, S. N. Pisharody, J. M. Murray, and P. H. Bucksbaum, Phys. Rev. A 71, 013407 (2005).

[12] C. Rangan, K. J. Schafer, and A. R. P. Rau, Phys. Rev. A 61, 053410 (2000).

[13] U. Fano and A. R. P. Rau, Atomic Collisions and Spectra (Academic, Orlando, 1986), Chap. 3, p. 5052.

[14] Eric J. Heller, J. Chem. Phys. 67, 3339 (1977).

[15] T. F. Gallagher, Rydberg Atoms (Cambridge University Press, Cambridge, U.K., 1994). 